\title{
Correlation between Respiratory Muscle Strength and Pulmonary Function with Respiratory Muscle Length Increase in Healthy Adults
}

\author{
Kyeongbong Lee ${ }^{a^{*}(\mathbb{D}}$

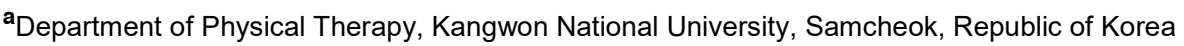

\begin{abstract}
Objective: The interest of clinicians is increasing due to the newly established medical insurance for pulmonary rehabilitation. Improvement of respiratory muscle strength and pulmonary function is an important factor in pulmonary rehabilitation, and this study aims to investigate the correlation between changes in respiratory muscle contraction thickness that can affect respiratory muscle strength and pulmonary function.
\end{abstract}

Design: Cross-sectional observational study.

Methods: Thirty-one subjects $($ male $=13$, female $=18$ ) participated in this study. The respiratory muscle strength was measured by dividing it into inspiratory/forced expiratory muscles, and the pulmonary function was measured by forced vital capacity (FVC), forced expiratory volume in 1 second (FEV1), and FEV1/FVC. To evaluate the respiratory muscle length increase, in resting and concentric contraction thickness of diaphragm, external/internal oblique, transverse abdominis, and rectus abdominis were measured by using ultrasonography.

Results: Inspiratory muscle strength showed a significant correlation with the length increase of the inspiratory muscle $(\mathrm{r}=0.368$ $\sim 0.521, \mathrm{p}<0.05$ ), and forced expiratory muscle strength showed a significant correlation with length increase of forced expiratory muscle $(\mathrm{r}=0.356 \sim 0.455, \mathrm{p}<0.05)$. However, pulmonary function was not correlated with the length increase of the respiratory muscle.

Conclusions: In this study, a correlation between respiratory muscle strength and respiratory muscle length increase was confirmed, but no correlation with the pulmonary function was found. It is considered that the respiratory muscle strength can be improved by increasing the respiratory muscle thickness through appropriate respiratory muscle training.

Key Words: Respiratory muscle, Pulmonary function test, Respiratory muscle thickness, Ultrasonography

\section{Introduction}

Rehabilitation breathing therapy for pulmonary diseases became reimbursable on December 1, 2016, there is a trend of increasing interest in clinical practice and researches. The features of pulmonary disease are airway obstruction or restriction of lung dilatation, even among pulmonary patients with similarly decreased lung capacity, a large difference in the degree of dyspnea and exercise capacity was observed.

The pulmonary function test measures lung volume, capacity, rates of flow, and the respiratory muscle strength test assesses the maximal inspiratory pressure (MIP), and the maximal expiratory pressure (MEP). It evaluates the inspiratory muscle such as the diaphragm and inspiratory muscle strength and forced expiratory muscle strength [1]. Because respiratory failure may occur due to respiratory muscle weakness, not the pulmonary disease[2, 3], it is needed to evaluate the respiratory muscle strength appropriately and to confirm its clinical significance.

One of the important methods of assessing the severity

Received: Sep 15, 2021 Revised: Oct 28, 2021 Accepted: Nov 2, 2021

Corresponding author: Kyeongbong Lee (ORCID https://orcid.org/0000-0003-4969-4018)

Department of Physical Therapy, Kangwon National University, 346, Hwangjo-gil, Dogye-eup, Samcheok-si, Gangwon-do, Republic of Korea Tel: +82-33-540-3375 Fax:+82-33-540-3379 E-mail: kblee@kangwon.ac.kr

This is an Open-Access article distributed under the terms of the Creative Commons Attribution Non-Commercial License (http://creativecommons.org/licenses/ by-nc/4.0) which permits unrestricted non-commercial use, distribution, and reproduction in any medium, provided the original work is properly cited. Copyright @ 2021 Korean Academy of Physical Therapy Rehabilitation Science 
of lung disease is the pulmonary function test. The most common method for assessing the severity is the pulmonary function test or the results of spirometry airflow. Pulmonary function tests are widely used as a method of evaluating the lung function of patients with pulmonary diseases in the clinical setting.

Respiratory musclesare divided into inspiratory muscle and forced expiratory muscle. During inhalation, the diaphragm contracts, so that its center moves downward and compresses the abdominal cavity. In addition, forced expiration can be achieved by contraction of the abdominal wall muscles[4], such as external oblique (EO), internal oblique (IO), transverse abdominis (TrA), and rectus abdominis (RA). Diaphragmatic ultrasound correlates with inspiratory muscle strength and pulmonary function [5], studies including the association between the thickness of the forced expiratory muscle and the respiratory muscle strength are insufficient.

Therefore, the purpose of this study is to investigate the association between respiratory muscle strength, pulmonary function and respiratory muscle thickness to determine which of the respiratory muscle strength and pulmonary function is related to respiratory muscle thickness.

\section{Methods}

\section{Participants}

The sample size of the present study was calculated by using the G-power version 3.1.9.4 (Universität Kiel, Kiel, Germany). The coefficient of determination $\rho^{2}=0.3$, correlation $\rho \mathrm{H} 1=0.54, \alpha$ error probability $=0.05$, and power $=0.08$, the number of study participants was 23 .

The subjects of this study were 14 males and 18 females' college students who were in their 20's at K University in $\mathrm{S}$ city. Inclusion criteria were: who have not experienced severe low back pain within the last 6 months, do not have systemic diseases that may affect the musculoskeletal system, do not have deformities or disorders that may affect the muscle thickness of the abdominal muscles, did not perform vigorous exercise within 48 hours before the experiment and do not take steroids and protein supplements. The exclusion criteria were: those with acute low back pain or persistent non-idiopathic low back pain, with a history of invasive procedures and surgeries on the lower back and abdomen, with limited lumbar movement due to spondylolisthesis, spondylolisthesis, and neurological diseases, and do not agree to participate in the experimental process. Since one subject had an invasive treatment of the lumbar region, the results of 31 subjects were analyzed. This study used a cross-sectional study design, 31 participants were engaged in the present study. All subjects understood the purpose and process of this study and participated voluntarily through written consent. This study was performed with the approval of the Institutional Review Board of Kangwon National University (KWNUIRB -2021-06-004-002). The written informed consentwas provided to all participants and the ethical principles of the Declaration of Helsinki were explained.

\section{Procedure}

This study used a cross-sectional study design. After the anthropometry of the participants was collected (Table 1), the respiratory muscle strength and pulmonary function were measured. The present study evaluated the MIP, peak inspiratory flow (PIF), forced inspiratory flow (FIF) 25-75\%, MEP, peak expiratory flow (PEF), and forced expiratory flow (FEF) $25-75 \%$. The forced vital capacity (FVC), FVC predicted, forced expiratory volume in 1 second (FEV1), FEV1 predicted, FEV1/FVC and $\mathrm{FEV} 1 / \mathrm{FVC}$ predicted were measured for pulmonary function

The respiratory muscles were classified as the forced expiratory and inspiratory muscles [2]. In this study, we investigated the thickness of the EO, IO, $\operatorname{TrA}$, and RA for forced expiratory muscle and diaphragm for inspiratory muscle. The respiratory muscle thickness was measured at rest, maximal expiration for the forced expiratory muscles, and maximal inspiration for the diaphragm.

Table 1. General characteristics of the participants. $(\mathrm{N}=31)$

\begin{tabular}{ll}
\hline & Value \\
\hline Age $(\mathrm{yrs})$ & $22.90 \quad(1.30)$ \\
Gender (male / female / total) & $13 / 18 / 31$ \\
Weight $(\mathrm{kg})$ & $60.77 \quad(10.06)$ \\
Height $(\mathrm{cm})$ & $165.68 \quad(7.73)$ \\
Body mass index $\left(\mathrm{kg} / \mathrm{m}^{2}\right)$ & $22.03(2.44)$ \\
\hline
\end{tabular}

Note: Values are presented as mean (SD) or number only. 


\section{Measurement}

\section{Respiratory muscle strength}

Respiratory muscle strength was measured by dividing the inspiratory muscle and the forced expiratory muscle[6]. Inspiratory muscle strength was measured MIP, PIF, and FIF $25-75 \%$, forced expiratory muscle was MEP, PEF, and FEF $25-75 \%$. The respiratory muscle strength was evaluated by using a digital spirometer (Pony FX MIP/MEP; COSMED Inc., Rome, Italy) according to the standards of the American Thoracic Society/European Respiratory Society[7]. Participants were seated in a chair without a backrest, and their feet were placed on the floor with the trunk and hip joints were kept at a $90^{\circ}$ angle. The inspiratory muscle strength was measured that the subject performed maximum inspiration strongly and rapidly in the state of maximum expiration while using a nose clipto prevent air leakage that may occur in the nose. The expiratory muscle strength was assessed that during the subject own breathing, he performed maximum inspiration with his range, the participants achieved maximum expiration as fast and strong as possible in maximum inspiration state with making sure that the mouthpiece of the equipment is as close to the lips as possible to prevent air leakage. Inspiratory muscle strength was measured by using the same device to inhale as quickly and strongly as possible (total lung capacity) at the end of vital capacity. This measurement process was performed three times by maintaining an interval of 15 minutes or more, and the highest value was selected as the result[8]. In thisstudy, the range of ICC of inspiratory and expiratory muscle strength was $0.939 \sim 0.982$.

\section{Pulmonary function}

The present study assessed the FVC, FVC predicted, FEV1, FEV1 predicted, FEV1. FVC and FEV1. FVC predicted for the pulmonary function. Pulmonary function was evaluated with the same spirometer and participant posture as the measurement procedure for respiratory muscle strength. A nose clip was usedfor reducing measurement error, and subjects were asked to exhale as quickly and strongly as possible (end of vital capacity) while inhaling as much as possible (total lung capacity) for more than 5 seconds [7]. Three measurements were taken with a break of 15 minutes or longer, and the highest value was used as the result. In this study, the range of ICC of pulmonary function was 0.931 0.952 .

\section{Respiratory muscle length increase}

The respiratory muscle thickness was measured using real-time B-mode ultrasonography was used with a 7.5 $\mathrm{MHz}$ linear transducer that PC-based ultrasonography MICRUS EXT-1H KIT and the muscle structure was analyzed through the Echo Wave II software (ver. 4.0.1, TELEMED UAB, Lithuania). To get ultrasound images of the diaphragm, the transducer was placed perpendicular to the point just above the lower intercostal angle and the anterior axillary line[9]. If the transducer is brought into contact with this position, the hyperechoic and hypoechoic layers of the pleura and peritoneum can be identified, and the distance between these two layers was measured as the thickness of the diaphragm. To measure the resting thickness of the diaphragm, the ultrasound image was verified at the end of expiration while the tidal volume was generated during respiration, and the image of contraction thickness was identified during maximum inspiration.

Among the respiratory muscle, the forced expiratory muscle thickness, EO, IO, TrA, and RA were measured in resting (at the end of expiration) and contraction (at maximal forced expiration). For the consistency of ultrasound imaging, the participants were in a crook-lying position and supports the pillow behind the knees to minimize the tension of the lumbar and abdominal region [10]. The subject's abdomen was exposed and ultrasound images were obtained using ultrasound gel on the subject's abdomen. To get a clear image, the transducer of the ultrasound device was placed on the anterior axillary line of the abdomen transversely and the thickness of the EO, IO, and TrA muscle was visualized between the 12th rib and the iliac crest[11]. At this position, the thickness of the EO, IO, and TrA muscles was imagined on one screen. The ultrasound images of the RA muscle were obtained at the same body position and the center of the transducer was placed vertically from the umbilicus, and the images were visualized at a point $2-3 \mathrm{~cm}$ above the umbilicus [11]. After the measurement, the resting and contraction 
thickness were analyzed through the Echo Wave II software (TELEMED). The thickness of the EO, IO, and $\operatorname{TrA}$ muscles was marked out by drawing a perpendicular guideline that was located $2.0 \mathrm{~cm}$ from the edge of the TrA muscle fascia junction and RA muscle thickness was obtained by drawing a vertical guideline that was located $2.0 \mathrm{~cm}$ from the edge muscle fascia junction of the RA muscle. The reliability of this measurement was generally excellent: ICCs ranged from 0.95 to 1.00 for intra-image reliability; 0.77 to 0.97 for inter-image reliability [10]. Respiratory muscle length was measured three times at intervals of 15 minutes or more, and the highest value was selected. In this study, the range of ICC of diaphragm thickness of the present study was $0.898 \sim 0.923$.

\section{Statistical analysis}

The collected data were analyzed using IBM SPSS Statistics (ver 23.0, IBM Co., USA). The general characteristics, respiratory muscle strength, pulmonary function, and respiratory muscle thickness were analyzed using descriptive analysis for the means, and standard deviations of the variables. Pearson correlation analysis was achieved to survey the correlation between respiratory muscle strength, pulmonary function, and respiratory muscle thickness. The values were considered statistically significant at $\mathrm{p}<0.05$.

\section{Results}

Table 2 demonstrates that the respiratory muscle strength, composed of inspiratory and expiratory muscles, and pulmonary function of the subjects. Table 3 shows the resting, contraction thickness, and length increase of the respiratory muscles. Table 4 reveals the correlation between respiratory muscle strength and the thickening ratio of the respiratory muscles. The MIP and PIF showed a significant correlation with diaphragm length increase. The length increase of the forced expiratory muscles demonstrated a tendency to show a correlation with expiratory muscle strength. Table 5 exhibits a correlation between pulmonary function and respiratory muscles length increase, no significant correlation was found.

Table 2. Respiratory muscle strength and pulmonary function of the participants. $\quad(\mathrm{N}=31)$

\begin{tabular}{lll}
\hline & \multicolumn{2}{l}{ Value } \\
\hline Respiratory muscle strength & & \\
$\quad$ Inspiratory muscles & & \\
MIP $\left(\mathrm{cmH}_{2} \mathrm{O}\right)$ & 67.35 & $(22.21)$ \\
$\mathrm{PIF}(\ell / \mathrm{s})$ & 5.30 & $(1.04)$ \\
FIF 25-75\% $(\ell / \mathrm{s})$ & 3.96 & $(1.14)$ \\
$\quad$ Forced expiratorymuscle & & \\
MEP $\left(\mathrm{cmH}{ }_{2} \mathrm{O}\right)$ & 71.26 & $(15.71)$ \\
PEF $(\ell / \mathrm{s})$ & 5.67 & $(1.60)$ \\
FEF 25-75\% $(\ell / \mathrm{s})$ & 4.18 & $(2.02)$ \\
Pulmonary function & & \\
FVC $(\ell)$ & 3.72 & $(0.81)$ \\
FVC predicted $(\%)$ & 85.45 & $(11.48)$ \\
FEV1 $(\ell)$ & 3.14 & $(0.75)$ \\
FEV1 predicted $(\%)$ & 84.19 & $(12.41)$ \\
FEV1/FVC $(\%)$ & 24.96 & $(7.96)$ \\
FEV1/FVC predicted $(\%)$ & 97.00 & $(9.59)$ \\
\hline
\end{tabular}

Note: Values are presented as mean (SD).

Abbreviations: MIP, maximal inspiratory pressure; PIF, peak inspiratory flow; FIF, forced inspiratory flow; MEP, maximal expiratory pressure; PEF, peak expiratory flow; FEF, forced expiratory flow; FVC, force vital capacity; FEV1, forced expiratory volume in one second.

Table 3. Respiratory muscle thickness and length increase of EO, IO, TrA, RA and diaphragm.

$(\mathrm{N}=31)$

\begin{tabular}{llll}
\hline & Resting & Contraction & Length increase \\
\hline EO $(\mathrm{mm})$ & $5.22(1.99)$ & $6.16(1.86)$ & $0.94(0.72)$ \\
IO $(\mathrm{mm})$ & $7.24(2.29)$ & $9.16(2.75)$ & $1.92(1.42)$ \\
TrA $(\mathrm{mm})$ & $2.96(1.23)$ & $5.05(1.57)$ & $2.09(1.10)$ \\
RA $(\mathrm{mm})$ & $6.96(2.06)$ & $8.80(2.15)$ & $6.13(1.91)$ \\
Diaphragm $(\mathrm{mm})$ & $2.67(0.97)$ & $13.62(3.74)$ & $10.95(4.06)$ \\
\hline
\end{tabular}

Note: Values are presented as mean (SD).

Abbreviations: EO, External oblique; IO, Internal oblique; TrA, transverse abdominis; RA, Rectus abdominis. 


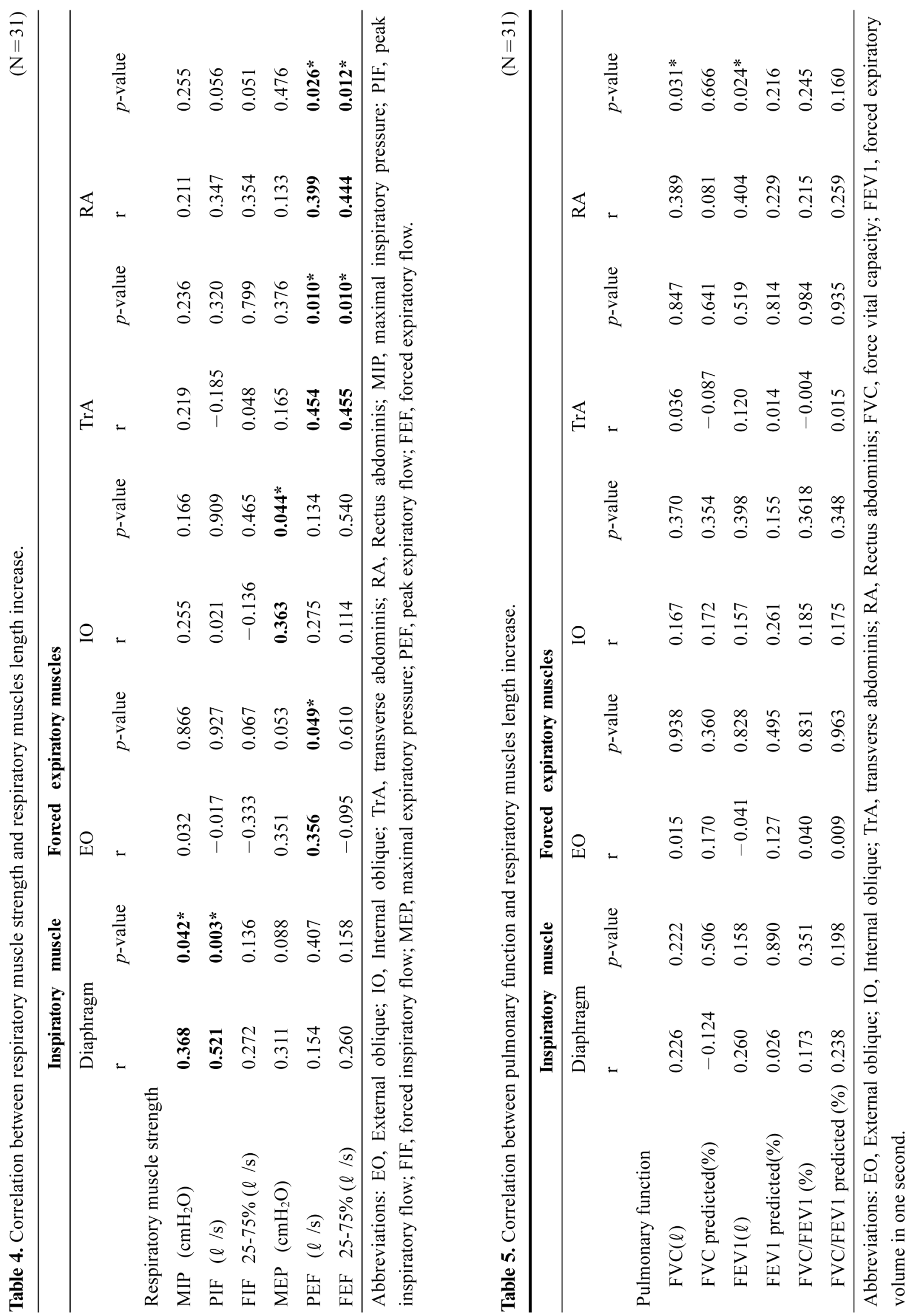




\section{Discussion}

The present study investigated the correlation between respiratory muscle strength and pulmonary function with respiratory muscle length increase. In respiratory muscle strength, inspiratory muscle strength showed a relationship with diaphragm thickening ratio and expiratory muscle strength demonstrated correlation with length increase of forced expiratory muscles. However, pulmonary function presented no relationship with respiratory muscle length increase.

Increased respiration is occurred during exercise due to an increase in both the depth and frequency of breathing. To efficiently increase the depth of respiration during exercise, more contractility of the inspiratory, forced expiratory and accessory muscles is required. The MIP and MEP are defined as indicators of inspiratory muscles and expiratory muscles, respectively [12]. The higher the inspiratory muscle strength, the more advantageous chest expansion is, so the lung volume can be further increased [13]. The vertical thoracic expansion occurs through the action of the diaphragm, and transverse expansion occurs through the movement of the rib cage and intercostal muscles. Thus, weakness of inspiratory and forced expiratory muscles can affect lung volume.

MIP and MEP are useful parameters for early detection of respiratory muscle dysfunction [14], Respiratory muscle weakness was significantly correlated with exercise capacity in healthy adults [15]. Exercise capacity refers to the patient's ability to perform functional activities and requires the integrated action of the cardiopulmonary and musculoskeletal systems. Pulmonary function in patients with the chronic obstructive pulmonary disease was significantly correlated with a decrease in chest expansion [16], and respiratory muscle strength in patients with heart failure was correlated with a decrease in exercise capacity [17]. Therefore, respiratory muscle strength is a factor that must be considered for functional recovery in patients with cardiopulmonary disease.

Pulmonary function refers to the activities of the lungs that enable a person to breathe well. The exchange of carbon dioxide and oxygen occurs in the lungs to provide the oxygen needed by body tissues. Carbon dioxide is generated as a by-product of energy production and it should be excreted from the body. However, if the lung function is reduced, this process cannot occur smoothly that causes various problems.

Chronic obstructive pulmonary disease (COPD) is characterized by decreased airflow, coughing, sputum production, and decreased physical activity [18]. In patients with COPD, the movement of the diaphragm is reduced due to the decrease of airflow, resulting in compensatory movement of respiratory muscles [19]. The pulmonary function test is one of the methods to evaluate the severity of lung diseases including COPD. Pulmonary function tests focus on lung volume, capacity, rates of flow, and gas exchange as opposed to a respiratory muscle strength test. Measuring pulmonary mechanics assesses the ability of the lungs to move huge volumes of air quickly through the airways to identify airway obstruction. The purpose of this study was to evaluate the respiratory muscle strength and pulmonary function in healthy adults and to investigate the correlation with the change in length increase from resting to concentric contraction thickness of the respiratory muscle.

The diaphragm acts as the main muscle in inspiration, and lateral abdominal muscles have pumping action, both activities should be performed efficiently. Ultrasound imaging has been established as a method for evaluating diaphragm thickness that can be measured in B-mode $[9,20]$. Patients with severe respiratory failure may require mechanical ventilation, it was reported that mechanical ventilation may have adverse effects on the thickness of respiratory muscles including the diaphragm and lateral abdominal muscles [21-23]. Abdominal muscles form the expiratory cavity of the trunk, EO, IO, and TrA constitute the lateral side, and RA institute the anterior area. Also, the diaphragm constitutes the superior area. Thus, proper contractility of these muscles is one of the essential factors for maintaining normal breathing.

Patients with respiratory or neuromuscular disease have reduced respiratory muscle strength and pulmonary function. Thus, some interventions improve these factors to improve the physical function and exercise capacity of the patients, it aims to improve aerobic capacity and physical function by increasing respiratory muscle strengthening exercise. To this end, muscle strengthening exercises are required in the intervention, which must be accompanied by the patient's ability to contract the respiratory muscles. In the results of this study, the 
diaphragm, one of the inspiratory muscles, showed a meaningful correlation with inspiratory muscle strength, and $\mathrm{EO}, \mathrm{IO}, \mathrm{TrA}$, and RA were confirmed to have a significant correlation with expiratory muscle strength. However, the pulmonary function, which measures the amount of flowing air, did not show a correlation with the respiratory muscle length increase. Since the pulmonary function measures airflow per unit time, lung volume, capacity, and rates of flow, it is considered that the correlation with respiratory muscle length increase, which can mean respiratory muscle strength, has not been confirmed.

This study has several limitations requiring future researches. First, subjects participating in this study were healthy adults, and the number of subjects was relatively small, the results of this study cannot be generalized in pulmonary disease patients. Second, the present study measured the muscle thickness for the concentric contraction thickness, which may not be a representative function for the respiratory muscles. Another limitation of this study, respiratory muscle strength and pulmonary function were evaluated in the sitting position, while respiratory muscle length increase was measured in the supine position. Respiratory muscle length increase can be reliably measured in the supine position since muscle thickness can be evaluated under conditions less affected by gravity. However, respiratory muscle strength and pulmonary function evaluated by a spirometer can be measured with high reliability.

\section{Conclusion}

Interests in pulmonary rehabilitation is increasing in clinical practice, and respiratory muscle strength and pulmonary function should be improved to progress the function of patients with respiratory diseases. In healthy adults, respiratory muscle length increase, which could affect respiratory muscle strength, showed a meaningful correlation, but pulmonary function was not. Improvement of respiratory muscle length increase is considered to be essential, and future studies on factors that may affect the progress of pulmonary function are needed.

\section{Conflict of Interest}

This study has been worked with the support of a research grant of Kangwon National University in 2020 .

\section{References}

1. Steier J, Kaul S, Seymour J, Jolley C, Rafferty G, Man W, et al. The value of multiple tests of respiratory muscle strength. Thorax. 2007;62(11):975-80.

2. Mier-Jedrzejowicz A, Brophy C, Moxham J, Green M. Assessment of Diaphragm Weakness1-3. Am Rev Respir Dis. 1988;137:877-83.

3. Davison A, Mulvey D. Idiopathic diaphragmatic weakness. Br Med J. 1992;304(6825):492.

4. Ratnovsky A, Elad D, Halpern P. Mechanics of respiratory muscles. Respir Physiol Neurobiol. 2008; 163(1-3):82-9.

5. Cardenas LZ, Santana PV, Caruso P, de Carvalho CRR, de Albuquerque ALP. Diaphragmatic ultrasound correlates with inspiratory muscle strength and pulmonary function in healthy subjects. Ultrasound Med Biol. 2018;44(4):786-93.

6. Vincken W, Ghezzo H, Cosio M. Maximal static respiratory pressures in adults: normal values and their relationship to determinants of respiratory function. Bull Eur Physiopathol Respir. 1987;23(5):435-9.

7. Nici L, Donner C, Wouters E, Zuwallack R, Ambrosino N, Bourbeau J, et al. American thoracic society/European respiratory society statement on pulmonary rehabilitation. Am J Respir Crit Care Med. 2006;173(12):1390-413.

8. Xiao Y, Luo M, Wang J, Luo H. Inspiratory muscle training for the recovery of function after stroke. Cochrane Database Syst Rev. 2012(5).

9. Harper CJ, Shahgholi L, Cieslak K, Hellyer NJ, Strommen JA, Boon AJ. Variability in diaphragm motion during normal breathing, assessed with B-mode ultrasound. J Orthop Sports Phys Ther. 2013;43(12):927-31.

10. Stetts DM, Freund JE, Allison SC, Carpenter G. A rehabilitative ultrasound imaging investigation of lateral abdominal muscle thickness in healthy aging adults. J Geriatr Phys Ther. 2009;32(2):16-22.

11. Tahan N, Khademi-Kalantari K, Mohseni-Bandpei 
MA, Mikaili S, Baghban AA, Jaberzadeh S. Measurement of superficial and deep abdominal muscle thickness: an ultrasonography study. J Physiol Anthropol. 2016;35(1):1-5.

12. Schoser B, Fong E, Geberhiwot T, Hughes D, Kissel JT, Madathil SC, et al. Maximum inspiratory pressure as a clinically meaningful trial endpoint for neuromuscular diseases: a comprehensive review of the literature. Orphanet J Rare Dis. 2017;12(1): $1-12$.

13. Lutfi MF. The physiological basis and clinical significance of lung volume measurements. Multidiscip Respir Med. 2017;12(1):1-12.

14. Chiang J, Mehta K, Amin R. Respiratory diagnostic tools in neuromuscular disease. Child. 2018;5(6):78.

15. Amann M. Pulmonary system limitations to endurance exercise performance in humans. Exp Physiol. 2012;97(3):311-8.

16. Kaneko H, Shiranita S, Horie J, Hayashi S. Reduced chest and abdominal wall mobility and their relationship to lung function, respiratory muscle strength, and exercise tolerance in subjects with COPD. Respir Care. 2016;61(11):1472-80.

17. Lin S-J, McElfresh J, Hall B, Bloom R, Farrell K. Inspiratory muscle training in patients with heart failure: a systematic review. Cardiopulm Phys Ther J. 2012;23(3):29.

18.Viegi G, Pistelli F, Sherrill D, Maio S, Baldacci S, Carrozzi L. Definition, epidemiology and natural history of COPD. Eur Respir J. 2007;30(5):993-1013.

19. De Andrade AD, Silva T, Vasconcelos H, Marcelino M, Rodrigues-Machado M, Galindo Filho V, et al. Inspiratory muscular activation during threshold ( therapy in elderly healthy and patients with COPD. J Electromyogr Kinesiol. 2005;15(6):631-9.

20. Boon AJ, Harper CJ, Ghahfarokhi LS, Strommen JA, Watson JC, Sorenson EJ. Two-dimensional ultrasound imaging of the diaphragm: quantitative values in normal subjects. Muscle Nerve. 2013; 47(6):884-9.

21. Levine S, Nguyen T, Taylor N, Friscia ME, Budak MT, Rothenberg P, et al. Rapid disuse atrophy of diaphragm fibers in mechanically ventilated humans. N Engl J Med. 2008;358(13):1327-35.

22. Van den Berg M, Hooijman PE, Beishuizen A, De Waard MC, Paul MA, Hartemink KJ, et al. Diaphragm atrophy and weakness in the absence of mitochondrial dysfunction in the critically ill. Am J Respir Crit Care Med. 2017;196(12):1544-58.

23. Misuri G, Colagrande S, Gorini M, Iandelli I, Mancini M, Duranti R, et al. In vivo ultrasound assessment of respiratory function of abdominal muscles in normal subjects. Eur Respir J. 1997;10(12): 2861-7. 